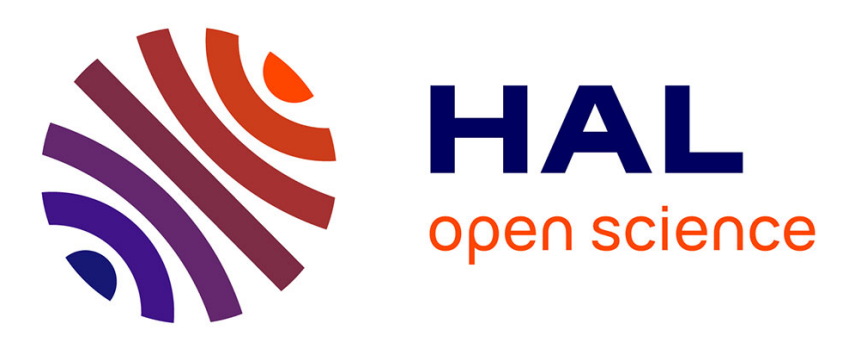

\title{
Elastic exciton-exciton scattering in photoexcited carbon nanotubes
}

D. T. Nguyen, C. Voisin, Ph. Roussignol, C. Roquelet, Jean-sébastien Lauret, Guillaume Cassabois

\section{- To cite this version:}

D. T. Nguyen, C. Voisin, Ph. Roussignol, C. Roquelet, Jean-sébastien Lauret, et al.. Elastic exciton-exciton scattering in photoexcited carbon nanotubes. Physical Review Letters, 2011, 107 (12), pp.127401. 10.1103/PhysRevLett.107.127401 . hal-00623859

\section{HAL Id: hal-00623859 \\ https://hal.science/hal-00623859}

Submitted on 19 Sep 2011

HAL is a multi-disciplinary open access archive for the deposit and dissemination of scientific research documents, whether they are published or not. The documents may come from teaching and research institutions in France or abroad, or from public or private research centers.
L'archive ouverte pluridisciplinaire HAL, est destinée au dépôt et à la diffusion de documents scientifiques de niveau recherche, publiés ou non, émanant des établissements d'enseignement et de recherche français ou étrangers, des laboratoires publics ou privés. 


\title{
Elastic exciton-exciton scattering in photoexcited carbon nanotubes
}

\author{
D. T. Nguyen ${ }^{1}$, C. Voisin ${ }^{1}$, Ph. Roussignol ${ }^{1}$, C. Roquelet ${ }^{2}$, J. S. Lauret ${ }^{2}$, and G. Cassabois ${ }^{1,3,4, *}$ \\ ${ }^{1}$ Laboratoire Pierre Aigrain, Ecole Normale Supérieure, UPMC, Université Paris Diderot, \\ CNRS UMR8551, 24 rue Lhomond 75231 Paris Cedex 5, France \\ ${ }^{2}$ Laboratoire de Photonique Quantique et Moléculaire de l'Ecole Normale Supérieure de Cachan, \\ 61 avenue du Président Wilson, 94235 Cachan Cedex, France \\ ${ }^{3}$ Université Montpellier 2, Laboratoire Charles Coulomb UMR5221, F-34095, Montpellier, France \\ ${ }^{4}$ CNRS, Laboratoire Charles Coulomb UMR5221, F-34095, Montpellier, France
}

(Dated: July 21, 2011)

\begin{abstract}
We report on original nonlinear spectral-hole burning experiments in single wall carbon nanotubes that bring evidence of pure dephasing induced by exciton-exciton scattering. We show that the collision-induced broadening in carbon nanotubes is controlled by exciton-exciton scattering as for Wannier excitons in inorganic semiconductors, while the population relaxation is driven by excitonexciton annihilation as for Frenkel excitons in organic materials. We demonstrate that this singular behaviour originates from the intrinsic one-dimensionality of excitons in carbon nanotubes, which display unique hybrid features of organic and inorganic systems.
\end{abstract}

PACS numbers: 78.67.Ch, 71.35.-y, 78.47.nd

Single wall carbon nanotubes (SWNTs) are rolled graphene sheets with nanometric radial quantum confinement, and the translation invariance along the tube axis makes them a reference one-dimensional (1D) system [1]. The one-dimensionality of carriers in SWNTs results in strong Coulomb interactions. The first striking signature is the non-perturbative binding of an electronhole pair into an exciton due to the attractive Coulomb potential [2]. The excitonic binding energy is found to be approximately one third of the single-particle gap $[3,4]$ and it opens the way for carbon-based optoelectronic devices operating at room-temperature [5-7]. Such a phenomenology resembles the binding of Frenkel excitons in organic materials. In these compounds, exciton-exciton collisions are known to control the population relaxation through an efficient exciton-exciton annihilation (EEA). This Auger process is one type of collisions where one exciton recombines and gives its energy to the second one, that is promoted to a high-energy state, resonant with the continuum of electron-hole pairs (Fig.1). This nonradiative recombination pathway plays a key role in the population relaxation of Frenkel excitons in organic materials, especially J-aggregates [8] and conjugated polymers [9]. On the contrary, for Wannier excitons in inorganic bulk semiconductors or quantum wells [10], manybody effects first manifest as elastic exciton-exciton scattering (EES) that redistributes the population within exciton bands (Fig.1) and leads to self-broadening of the excitonic transition. In the prospect of applications, the understanding and the control of the excitonic nonlinearities appears as a central issue for the development of optoelectronic devices, such as lasers, switches or quantum light sources $[5-10]$.

In SWNTs, several time-resolved experiments have shown evidence for efficient EEA that drives the population dynamics even at low exciton density [11, 12]. This observation supports the analogy with organic materials and further raises the question of the excitonic decoherence in SWNTs. In organic compounds, the decoherence induced by exciton-exciton collisions comes from the population relaxation due to EEA, whereas in inorganic systems it consists in pure dephasing by EES (the pure dephasing being usually characterized by the $T_{2}^{\star}$ time given by $1 / T_{2}=1 / 2 T_{1}+1 / T_{2}^{\star}$ where $T_{1}$ and $T_{2}$ are the population and coherence relaxation times, respectively). In inorganic semiconductors, the EEA process also affects the relaxation dynamics but at very high excitation densities. On the contrary, the lack of evidence for EES in organic materials is in very high contrast to inorganic semiconductors, as pointed out in Ref.[13].

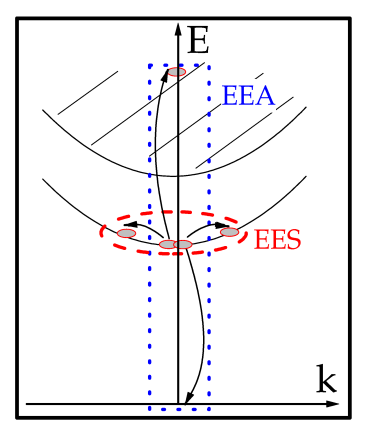

FIG. 1. Exciton energy dispersion and schematic representation of the Auger recombination process of exciton-exciton annihilation (EEA, in blue), and the purely dephasing mechanism of elastic exciton-exciton scattering (EES, in red). The dashed lines delimitate the exciton paths in k-space for EEA and EES.

Here, we show that the collision-induced broadening of excitons in carbon nanotubes is controlled by EES as for Wannier excitons in inorganic semiconductors, while the population relaxation is driven by EEA as for Frenkel excitons in organic materials. Our measurements are performed by means of an original technique of spectral 
hole-burning spectroscopy, that allows us to explore the exciton decoherence over three decades of excitation density. We observe that the collision-induced broadening is not limited by EEA and we demonstrate that the major contribution consists in pure dephasing by EES. Our interpretation is supported by theoretical calculations of the excitonic nonlinearities in SWNTs, and a quantitative agreement with our experimental results is obtained by calculation of EES based on a nonequilibrium formalism within the second Born approximation.

We have studied SWNTs synthetized by laser ablation of a doped graphite target and purified by ultrasonic and chemical treatment [14]. Semiconducting SWNTs contribute to the optical absorption by inhomogeneously broadened lines centered at 0.75 and $1.3 \mathrm{eV}$, respectively [14]. Metallic SWNTs absorb at higher energies around $1.9 \mathrm{eV}$ [14]. Our work is focused on the fundamental excitonic transition in semiconducting SWNTs absorbing around $0.75 \mathrm{eV}$ (Fig.2, inset). In the prospect of evidencing the intrinsic properties of $1 \mathrm{D}$ excitons, we have tried to suppress the influence of external non-radiative relaxation processes as much as possible. Therefore, the SWNTs are first isolated by the micelle-wrapping technique $[15,16]$ which allows the observation of a bright photoluminescence signal, as shown by the bottom curve in the inset of Fig.2. The individual SWNTs are further embedded in a gelatin matrix following the procedure described in Ref.[17], in order to perform measurements at cryogenic temperatures thus minimizing the contribution of exciton-phonon dephasing.

Nonlinear spectral hole-burning experiments were performed around $0.8 \mathrm{eV}(\lambda \sim 1.55 \mu \mathrm{m})$ [18]. Our study under cw-excitation differs from previous nonlinear optical measurements which focused on the ultrafast coherent nonlinear response and its specific aspects in the context of 1D excitons in SWNTs [19]. In our spectrallyresolved pump-probe differential transmission measurements, a narrow pump laser centered at $0.8 \mathrm{eV}$ (indicated by the vertical arrow in the inset of Fig.2) partially saturates the absorption of the SWNTs that are in resonance. The corresponding sub-ensemble of SWNTs reduces the overall absorption of the sample in a spectral window determined by the homogeneous linewidth $\Gamma$. The photoinduced bleaching of the SWNTs sample is detected by recording the change of transmission of a tunable probe laser. A typical spectrum of the pump-induced differential transmission $\Delta T / T$ of the probe laser is displayed in Fig.2. The nonlinear signal exhibits a pronounced bleaching with an amplitude of $3 \times 10^{-4}$ and a full width at half maximum (FWHM) of $6 \mathrm{meV}$, that is attributed to the excitonic hole-burning signal of the SWNTs. This component is superimposed on a negative signal, which corresponds to a residual non-resonant photo-absorption that is also detected at very large detuning with the pump laser.

The analysis of absorption saturation measurements

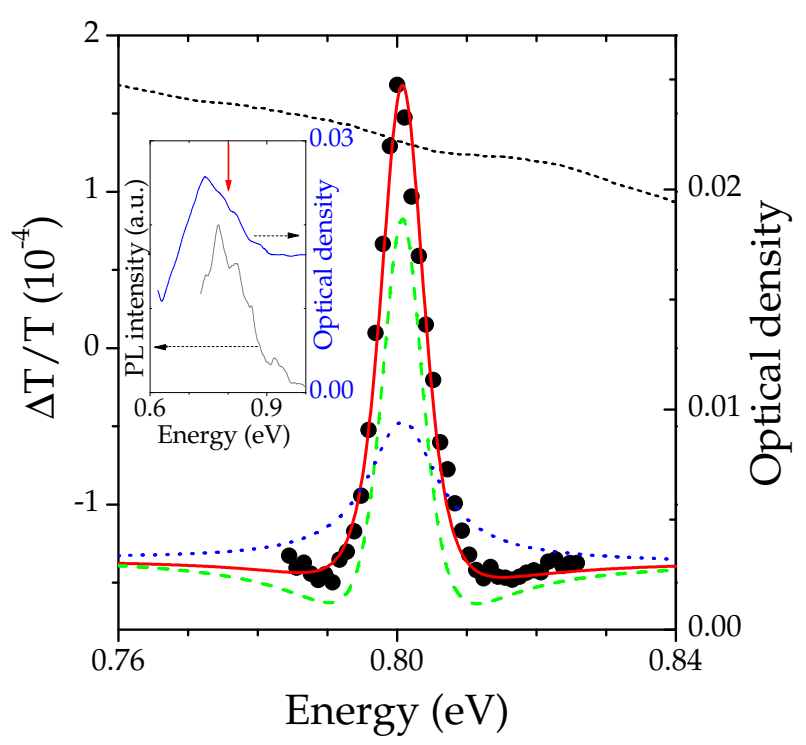

FIG. 2. (Left axis) Differential transmission $\Delta T / T$ of the probe laser at $10 \mathrm{~K}$ with a pump laser of $4 \mathrm{~kW} . \mathrm{cm}^{-2}$ at 0.8 $\mathrm{eV}$, as a function of the probe laser energy. The red curve is the sum of the differential transmissions $\Delta T / T$ calculated for $\Delta \Gamma=0$ (blue line) and $\Delta f=0$ (green line). (Right axis) Optical density (dashed line) spectrum obtained by linear absorption spectroscopy of the carbon nanotubes gelatine sample. Inset: (right axis) optical density displayed on a large spectral range. The red arrow indicates the pump laser energy of $0.8 \mathrm{eV}$ used for hole-burning measurements in semiconducting carbon nanotubes. Photoluminescence intensity (left axis) under non-resonant excitation at $2.33 \mathrm{eV}$.

obeys different rules depending on the fermionic or bosonic statistics of the elementary excitation of the system. In the case of excitonic transitions, the saturation of absorption is not due to a redistribution of an electronic population among the ground and excited states as in atomic systems, but to a change of the oscillator strength $f$ and homogeneous linewidth $\Gamma$ of the excitonic transition on raising the exciton density, as described in Ref.[20]. When analyzing our measurements as a function of pump power (see details of the technical aspects in the supplemental material [21]), we take advantage of the different signatures of collision-induced broadening $(\Delta \Gamma)$ and oscillator strength renormalization $(\Delta f)$ to disentangle these two components and extract their contribution to the nonlinear response (Fig.2, green and blue dashed lines). The result of our data analysis is summarized in Fig.3(a) where we display the relative variations of the homogeneous linewidth $\Delta \Gamma / \Gamma$ (full circles) and oscillator strength $-\Delta f / f$ (open squares) as a function of the pump power. Although both components are required to fit the width and amplitude of our differential transmission signal (Fig.2), we find that $\Delta \Gamma / \Gamma$ provides the major nonlinearity, thus leading to a good relative precision in its estimation but to a larger uncertainty for the 
minor contribution of $-\Delta f / f$, as seen in Fig.3(a). In the prospect of the quantitative interpretation of exciton dephasing in SWNTs, we finally stress that the recording of both collision-induced broadening and oscillator strength renormalization appears to be a fundamental feature for extracting the relative contributions of EEA and EES, which are at the heart of this paper.
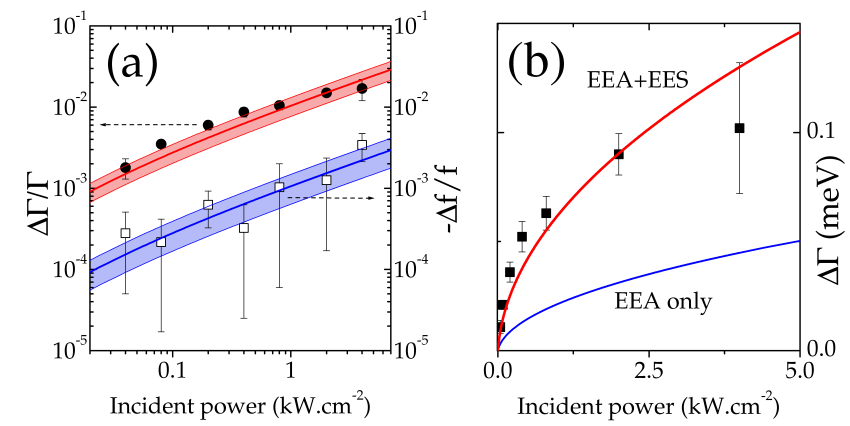

FIG. 3. (a) Relative variations of the homogeneous linewidth $\Delta \Gamma / \Gamma$ (full circles), and oscillator strength $-\Delta f / f$ (open squares), at 10K. Data (symbols) and theoretical fits (solid lines) are plotted as a function of the incident power of the pump laser. The fitting parameter is the exciton Bohr radius $a_{B}=5 \mathrm{~nm}$, and the shaded areas correspond to the tolerance of $\pm 2 \mathrm{~nm}$ in its estimation. (b) Homogeneous linewidth variations $\Delta \Gamma$ as a function of pump power. Theoretical fits: EEA only (blue line), EEA+EES (red line).

We have developped a theoretical model (described below) that leads to a global quantitative interpretation of our measurements with the exciton Bohr radius $a_{B}$ as the only free parameter. Its estimation is strongly constrained by the simultaneous comparison of $-\Delta f / f$ and $\Delta \Gamma / \Gamma$ with our theoretical predictions, and we find $a_{B}=5 \pm 2 \mathrm{~nm}$ (Fig.3(a)). This value is consistent with the calculation of the Bohr radius for SWNTs with a diameter of $1.2 \mathrm{~nm}$ (i.e. an excitonic transition around 0.8 $\mathrm{eV}$ ) inside a micelle structure $[2,22,23]$. In fact, our study reveals the original phenomenology of $1 \mathrm{D}$ excitons in SWNTs, where the exciton Bohr radius is of the order of magnitude of the nanotube diameter. This results in physical properties usually separately observed in inorganic and organic materials, as detailed below.

In the framework of the model developped by SchmittRink et al. for excitonic nonlinearities in 2D quantum wells [20], we first discuss the analysis of the oscillator strength renormalization by using the expression $\frac{\Delta f}{f}=-\frac{n}{n_{s}}$, which is valid for small perturbation $\left(n \ll n_{s}\right)$, where $n$ is the exciton density, and $n_{s}$ the saturation density. The exciton density $n$ is fixed by the characteristic parameters of the population relaxation in SWNTs. Its estimation for a given pump power is a key factor and requires a careful analysis (see supplemental material [21]). The saturation density $n_{s}$ is fixed by two mechanisms, which are both related to the exclusion principle: phase space filling corresponding to the conduction and valence band filling under generation of excitons, and exciton wave-function renormalization due to the screening of the electron-hole interaction in the presence of other carriers [20]. Let us arbitrarily define the Mott density $n_{M}$ by $1 / \pi a_{B}^{2}$ and $1 / 2 a_{B}$ for $2 \mathrm{D}$ and $1 \mathrm{D}$ excitons respectively, with $a_{B}$ the exciton Bohr radius. In $2 \mathrm{D}$ quantum wells, Schmitt-Rink et al. showed that $1 / n_{s}=\left(C_{P S F}+C_{E W R}\right) / n_{M}$ where $C_{P S F}=32 / 7$ and $C_{E W R} \sim 3.94$ are the numerical coefficients associated to phase space filling and exciton wave-function renormalization, respectively. We have extended the theoretical treatment of Ref.[20] to the case of $1 \mathrm{D}$ excitons. We find $C_{P S F}=3 / 2$ and $C_{E W R}=1 / 4$, thus showing that there is no dramatic dimensional effect on these coefficients, and that phase space filling predominates when increasing the confinement.

The variations of $\Delta \Gamma / \Gamma$ with pump power characterize the effect of collision-induced broadening (we checked that there is no thermal effect due to the pump). The first striking feature is the sublinearity of the collision-induced broadening as a function of the excitation density, as illustrated by the linear-linear plot of $\Delta \Gamma$ in Fig.3(b). Under the assumption [21] of a linear dependence of the collision-induced broadening on the exciton density, our data indicate that the stationary exciton density does not depend linearly on the pump fluence (so that $\Delta f / f$ is also sublinear with the pump power). This phenomenology is the manifestation of the interband Auger process of EEA depicted in Fig.1. Several groups have brought evidence by means of time-resolved experiments that this mechanism controls the relaxation dynamics of the exciton population in SWNTs $[11,12]$. In our measurements performed under cw-excitation, EEA results in a sublinear variation of the stationary exciton density with the pump power [21]. The predominance of EEA in the population relaxation is in fact not specific to excitons in SWNTs and is a general trend for Frenkel excitons in conjugated polymers or J-aggregates $[8,9]$.

We show now that this similarity between organic materials and the pure carbon-based nanostructures formed by SWNTs breaks down for the coherence relaxation induced by exciton-exciton collisions. In Fig.3(b) we have displayed in solid blue line $\Delta \Gamma_{E E A}$, the contribution of EEA to collision-induced broadening given by $2 \hbar A n$ where $A$ is the Auger annihilation coefficient defined in Ref.[24]. Since EEA drives the population relaxation, it also induces coherence relaxation but it is not sufficient to account for the excitation power dependence of the spectral broadening. This issue is totally unaddressed in the literature, and the quantitative interpretation of our experimental results presented in Fig.3 is achieved by taking into account EES. This intraband mechanism corresponds to the exciton-exciton collisions that lead to pure dephasing by quasi-elastic exciton scattering within the fundamental exciton band (Fig.1). The calculation of the self-broadening due to EES requires the theoretical 
framework of the second Born approximation which was successfully applied for $3 \mathrm{D}$ and $2 \mathrm{D}$ excitons $[25,26]$. This theory is also mandatory in $1 \mathrm{D}$ because of the divergence of the exciton density of states at the band edge, reminiscent of Van Hove singularities. In the framework of the regularization procedure of the Coulomb interaction based on a contact potential [24], we find:

$$
\Delta \Gamma_{E E S}=E_{b} n \frac{2 \hbar^{2}}{\mu a_{B}} \int_{-\infty}^{+\infty} d \widetilde{q}|I(\widetilde{q})|^{2} \frac{2 \Gamma^{\prime}}{\pi} \frac{1}{\Lambda^{2} \widetilde{q}^{4}+\left(2 \Gamma^{\prime}\right)^{2}}
$$

where $\Gamma^{\prime}=\Gamma+\Delta \Gamma_{E E S}$ with $\Gamma=6 \mathrm{meV}$ the homogeneous linewidth without exciton-exciton interactions, $\mu$ the excitonic reduced mass, $\Lambda=\hbar^{2} /\left(m_{e}+m_{h}\right) a_{B}^{2}$ with $m_{e}$ and $m_{h}$ the electron and hole masses equal to $0.1 m_{0}[1], I(\widetilde{q})$ the matrix element of fermion-fermion exchange interactions given by $\frac{16}{\left(\widetilde{q}^{2}+4\right)^{2}}-\frac{6}{\left(\widetilde{q}^{2}+4\right)\left(\widetilde{q}^{2}+1\right)}$, and $E_{b}$ the exciton binding energy of $250 \mathrm{meV}[2-4]$.

The competition between EEA and EES is at the heart of the physics governing the collision-induced broadening in SWNTs. The efficiency of these excitonic collision processes is conveniently characterized by the dimensionless parameters $\xi_{E E A}$ and $\xi_{E E S}$, where the collisioninduced broadening is expressed as $\Delta \Gamma=E_{b} \xi \frac{n}{n_{M}}$, with $\xi=\xi_{E E A}+\xi_{E E S}$. The linear dependence of $\Delta \Gamma$ with $E_{b}$ stems from the Coulomb nature of exciton-exciton interactions which impose the typical energy scale of electronic correlations between excitons. Space filling is expressed by the ratio $n / n_{M}$. The numerical values of $\xi_{E E A}$ and $\xi_{E E S}$ depend on the Auger annihilation rate $A$, exciton binding energy $E_{b}$ and exciton Bohr radius $a_{B}$ (see above). Our fitting procedure consists in analyzing our whole set of data with $a_{B}$ as the only free parameter, other quantities being documented in the literature [21]. The estimation of $a_{B}=5 \pm 2 \mathrm{~nm}$ thus leads to the values $\xi_{E E A}=0.14$ and $\xi_{E E S}=0.27$.

\begin{tabular}{c|cccl}
$\mathrm{d}$ & $\xi_{E E S}$ & $E_{b}(\mathrm{meV})$ & $E_{b} \xi_{E E S}(\mathrm{meV})$ & Ref. \\
\hline 3 & 6.3 & 4 & 25 & {$[27]$} \\
2 & 0.47 & 8.5 & 4 & {$[28]$} \\
1 & 0.27 & 250 & 68 & this study
\end{tabular}

TABLE I. Collision-induced broadening parameters as a function of exciton dimensionality $d . E_{b}$ is the exciton binding energy, and $\xi_{E E S}$ a dimensionless parameter.
While the exciton binding energy $E_{b}$ comprises the modification of the electronic Coulomb correlations, the $\xi_{E E S}$ factor contains the information on the density of final states in the scattering process, which is also strongly dimension dependent. From Table 1 where we observe that the $\xi_{E E S}$ factors decrease on reducing the exciton dimensionality, we conclude that the strong efficiency of EES in SWNTs arises from the exciton binding energy which is indeed two orders of magnitude larger than for $3 \mathrm{D}$ or $2 \mathrm{D}$ excitons, and imposes the overall increase of the collision-induced broadening with confinement, since $E_{b} \xi_{E E S}$ reaches its highest value in 1D (Table 1).

As far as the interplay between EEA and EES is concerned, an important parameter appears to be the ratio $E_{b} / E_{g}$ where $E_{g}$ is the single-particle energy gap. As pointed out in Ref.[24], the efficiency of EEA scales like $E_{b} / E_{g}$, and so does $\xi_{E E A}$, thus explaining the predominance of EEA in strongly bound Frenkel excitons in organic materials. We therefore conclude that SWNTs characterized by an exciton Bohr radius $a_{B}=5 \pm 2 \mathrm{~nm}$ correspond to an intermediate confinement regime between Wannier and Frenkel excitons, at the frontier of inorganic and organic materials.

In summary, we have shown that the collision-induced broadening of excitons in carbon nanotubes is controlled by EES as for Wannier excitons in inorganic semiconductors, while the population relaxation is driven by EEA as for Frenkel excitons in organic materials. Our study reveals the striking hybrid properties of $1 \mathrm{D}$ excitons in carbon nanotubes at the crossover of molecular systems and solid-state nanostructures. Our work motivates the exploration of possible scaling laws as a function of the nanotube diameter and the investigation of the limit cases of excitons in nanotubes of very large diameter, corresponding to graphene, and vanishing diameter, such as $\pi$-conjugated polymers.

We thank C. Ciuti and T. Ando for helpful discussions. This work was financially supported by the CNano IdF contract TAIGA and the ANR agency-contract $B O N A F O$.

*e-mail: Guillaume.Cassabois@univ-montp2.fr
[1] R. Saito, G., Dresselhaus and M.S. Dresselhaus, M. S., in Physical properties of carbon nanotubes, Imperial College Press (1998).

[2] V. Perebeinos et al., Phys. Rev. Lett. 92, 257402 (2004).

[3] F. Wang et al., Science 308, 838 (2005).

[4] J. Maultzsch et al., Phys. Rev. B 72, 241402(R) (2005).

[5] E. Gaufres et al., Appl. Phys. Lett. 96, 231105 (2010).

[6] A. Högele et al., Phys. Rev. Lett. 100, 217401 (2008).

[7] R. Matsunaga et al., Phys. Rev. Lett. 106, 037404 (2011).
[8] G.M. Akselrod et al., Phys. Rev. B 82, 113106 (2010).

[9] I.B. Martini et al., Phys. Rev. B 69, 035204 (2004).

[10] J. Shah, in Ultrafast spectroscopy of semiconductors and semiconductor nanostructures, Springer, Berlin (1999).

[11] F. Wang et al., Phys. Rev. B 70, 241403(R) (2004).

[12] Y-Z. Ma et al., Phys. Rev. Lett. 94, 157402 (2005).

[13] M. Shimizu et al., Phys. Rev. B 64, 115417 (2001).

[14] O. Jost et al., Appl. Phys. Lett. 75, 2217 (1999).

[15] M. O'Connell et al., Science 297, 593 (2002).

[16] J.S. Lauret et al., Physica E 21, 1057 (2004). 
[17] S. Berger et al., Nano Lett. 7, 398 (2007).

[18] D.C. Trung et al., Appl. Phys. Lett. 97, 061903 (2010).

[19] A. Madea et al., J. Phys. Soc. Jpn. 75, 043709 (2006); S. Tao et al., Phys. Rev. B 80, 201405(R) (2009); A. Madea et al., Phys. Rev. Lett. 94, 047404 (2005).

[20] S. Schmitt-Rink et al., Phys. Rev. B 32, 6601 (1985).

[21] See supplemental material for details on data analysis.

[22] T.G. Pedersen, Phys. Rev. B 67, 073401 (2003).

[23] D. Kammerlander et al., Phys. Rev. Lett. 99, 126806
(2007).

[24] F. Wang et al., Phys. Rev. B 73, 245424 (2006).

[25] F. Boldt et al., Phys. Status Solidi B 130, 675 (1985).

[26] C. Ciuti et al., Phys. Rev. B 58, 7926 (1998).

[27] L. Schultheis et al., Phys. Rev. Lett. 57, 1635 (1986).

[28] A. Honold et al., Phys. Rev. B 40, 6442 (1989). 